\title{
Research on the Fatigue Performance of TC6 Compressor Blade under the CCF Effect
}

\author{
Zhang Yakui $\mathbb{D}^{1}$ and Guo Shuxiang ${ }^{2}$ \\ ${ }^{1}$ Aeronautics and Astronautics Engineering College, Air Force Engineering University, Xi'an 710038, China \\ ${ }^{2}$ Department of Mathematics and Physics College, Air Force Engineering University, Xi'an 710038, China
}

Correspondence should be addressed to Zhang Yakui; 379610749@qq.com

Received 23 June 2018; Revised 9 August 2018; Accepted 15 August 2018; Published 24 September 2018

Academic Editor: Giovanni Delibra

Copyright ( $) 2018$ Zhang Yakui and Guo Shuxiang. This is an open access article distributed under the Creative Commons Attribution License, which permits unrestricted use, distribution, and reproduction in any medium, provided the original work is properly cited.

\begin{abstract}
This paper studied the influence of high and low combined fatigue (CCF) on compressor blade fatigue performance. We investigated the coupling between low cycle fatigue (LCF) loading from centrifugal force with high cycle fatigue (HCF) loading from vibration and determined the blade disc vibration frequency using static analysis at maximum rotational speed. We designed and constructed a combined fatigue test rig, and CCF tests were performed on a TC6 compressor blade to analyze fatigue life characteristics. Results showed that CCF could significantly shorten blade life compared with pure LCF and that larger HCF caused more significant fatigue life reduction. Fatigue source characteristics and CCF fracture appearance were observed and analyzed using a scanning electron microscope (SEM).
\end{abstract}

\section{Introduction}

Aircraft engine blade fatigue failure is a typical failure mode that drastically affects engine reliability and flight performance [1-7]. During operation, aircraft engines always endure low-amplitude, high-frequency vibration combined with high-level, low-frequency stress causing a combined fatigue effect. Modern compressor blades are designed to withstand stresses generated at resonance, but it is important to ensure blade integrity and life not only under HCF but also when superimposed with other damage mechanisms, such as LCF, which is inevitable in the compressor engine environment [8]. Most previous fatigue behavior studies of structural materials considered either LCF or HCF loading alone, although engineering components experience varying load history [9]. Pure LCF and HCF fatigue life predictions have large deviations compared to observation and did not reflect actual operational damage processes.

Several CCF studies have been reported in the last few decades. Fuchs et al. [10] were among the first to study CCF fatigue problems. The US Air Force subsequently acknowledged the CCF importance and designed experiments to explore interactions between LCF and HCF, showing that HCF fatigue limit increased under CCF [11]. Moshier et al. [12] also obtained similar conclusions and showed that HCF fatigue limit was higher under CCF than pure HCF conditions. Various foreign studies have also shown that LCF life is significantly shortened under CCF effect. Dungey and Bowen [13] confirmed this conclusion and tested titanium alloys under $\mathrm{CCF}$ and showed that the HCF life negligibly effected when the cycles exceeded $10^{5}$. Wang et al. [14] conducted the CCF test to study the extrapolation of turbine disc. Schweizer et al. [15] studied the mechanism and modeled fatigue crack growth under CCF. Both studies found that centrifugal and thermal stresses caused LCF turbine disc failures, whereas aerodynamically induced vibration stresses caused HCF failures. Powell et al. [16] systematically studied the influence of the number of HCF cycles per LCF cycle on fatigue crack growth. Gelmedin and Lang [17] studied IN 713C fatigue behaviors under LCF, HCF, and CCF conditions and found that, compared with the pure LCF loading, the superimposed HCF could dramatically shorten blade lifetime if its amplitude was high enough. Many studies have considered the same problems of the combined fatigue effects, researching that 
TABLE 1: Material properties of the studied material.

\begin{tabular}{lcccccc}
\hline $\begin{array}{l}\text { Property } \\
20^{\circ} \mathrm{C}\end{array}$ & $\begin{array}{c}\sigma_{b} \\
(\mathrm{MPa})\end{array}$ & $\begin{array}{c}\sigma_{0.2} \\
(\mathrm{MPa})\end{array}$ & $\psi$ & $\begin{array}{c}E \\
(\mathrm{GPa})\end{array}$ & $\begin{array}{c}\text { Poisson } \\
\text { ratio }\end{array}$ & $\begin{array}{c}\text { Density } \\
\rho / \mathrm{kg} / \mathrm{m}^{3}\end{array}$ \\
\hline & 980 & 1005 & 0.25 & 125 & 0.3 & 4450 \\
\hline
\end{tabular}

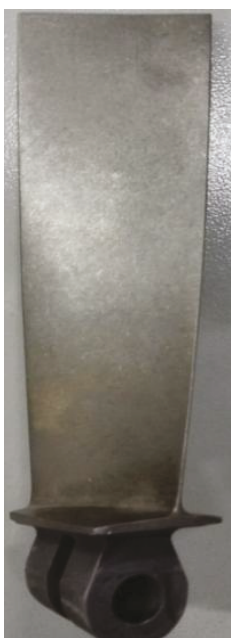

(a) Solid blade

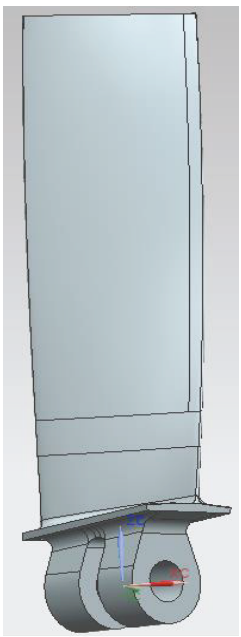

(b) UG model
Figure 1: Blade model.

combined fatigue life is more important and complex, and most studies considered turbine blades with few considering compressor blades [18-22].

In this paper, we first established a finite element model for the compressor blade and experimentally verified model accuracy by measuring the natural frequency of the rootretaining blade using impulse the hammering method. Static and vibration analyses of the blade disc system were then performed to obtain CCF test conditions and identify LCF load and HCF vibration frequency that produced maximum stress in the blade hazardous location. We formulated a CCF load spectrum based on those studies and realized a combined fatigue test rig. Fracture characteristics under CCF were analyzed to verify test design rationality. Last, the blades were also tested under pure LCF as well as CCF showing that the superimposition of vibration stress can lead to a significant reduction of LCF life.

\section{Experimental Design}

\subsection{Finite Element Analysis}

2.1.1. TC6 Characteristics. The most common blade material for high-pressure compressors employed in engines is TC6, strengthened martensitic thermally stable stainless steel with excellent overall mechanical properties, high thermal strength, and good stress corrosion resistance, hence very suitable for aeroengine blades. Table 1 shows the material properties $[23,24]$.

2.1.2. Blade Model Building. Figure 1 shows the threedimensional blade model built in Unigraphics NX software
TABLE 2: Blade geometric description.

\begin{tabular}{lc}
\hline Blade & Geometric size $(\mathrm{mm})$ \\
\hline High & 184.5 \\
Wide & 44.5 \\
Inner diameter & 11.5 \\
Outer diameter & 24.3 \\
Earring thickness & 12.5 \\
Inner surface spacing & 6.7 \\
\hline
\end{tabular}

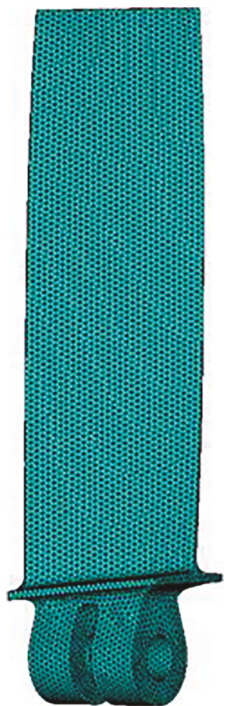

Figure 2: Blade mesh model.

TABLE 3: Blade model simulation results.

\begin{tabular}{lcc}
\hline Order & Natural frequency $(\mathrm{Hz})$ & Vibration type \\
\hline The first order & 251.68 & First bend \\
The second order & 873.58 & First scroll \\
The third order & 1400.8 & Second bend \\
The fourth order & 1967.9 & Second bend torsion \\
\hline
\end{tabular}

following its design drawings, and Table 2 shows the geometric parameters.

We meshed the blade model using Solid187 10-node tetrahedron solid units (Solid187 is a higher order 3D 10-node solid structural element). The mesh size was set to $1 \mathrm{~mm}$, as shown in Figure 2.

After establishing the blade finite element analysis (FEA) model, we calculated the blade model and defined boundary conditions such that all nodes on the inner ring of the two pinholes were fixed and constrained. We used Block Lanczos, an eigenvalue solver based on the Lanczos algorithm that is widely used in structural mode calculation, to simulate the first-four natural vibration frequency orders. The results were observed using the general post-processor POST1, as shown in Table 3.

The second-order natural blade vibration frequency $(873.58 \mathrm{~Hz})$ was much larger than the first-order frequency $(251.68 \mathrm{~Hz})$. The considered engine had 11,320 rpm design 


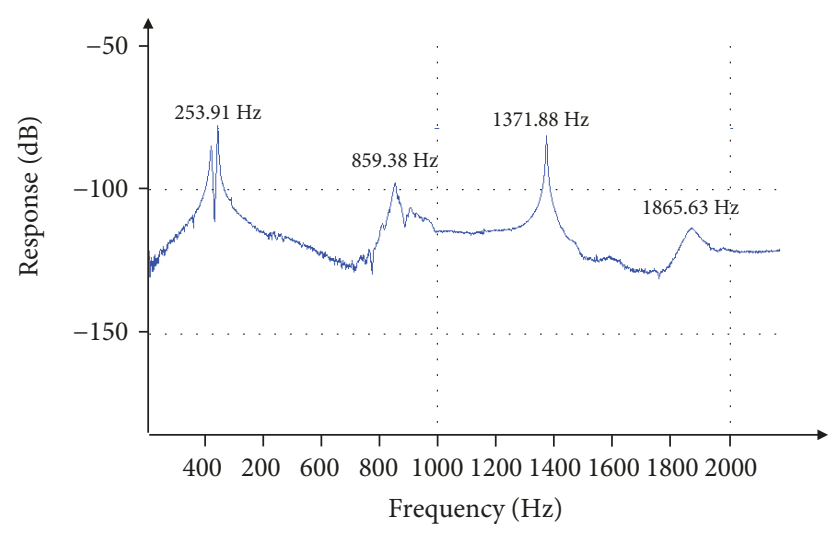

FIGURE 3: Frequency response of a1.

TABLE 4: Natural vibration frequencies of the blades.

\begin{tabular}{lcccc}
\hline $\begin{array}{l}\text { Blade } \\
\text { number }\end{array}$ & $\begin{array}{c}\text { The first } \\
\text { order }\end{array}$ & $\begin{array}{c}\text { Nhe second } \\
\text { order }\end{array}$ & $\begin{array}{c}\text { The third } \\
\text { order }\end{array}$ & $\begin{array}{c}\text { The fourth } \\
\text { order }\end{array}$ \\
\hline a1 & 253.91 & 859.38 & 1371.88 & 1865.63 \\
a2 & 243.81 & 840.62 & 1312.51 & 1824.32 \\
a3 & 247.72 & 861.69 & 1347.71 & 1851.24 \\
a4 & 247.14 & 853.13 & 1342.24 & 1849.08 \\
a5 & 249.21 & 852.27 & 1353.89 & 1847.85 \\
a6 & 253.88 & 868.83 & 1400.77 & 1868.21 \\
\hline
\end{tabular}

speeds, hence periodic excitation generated by the engine rotor on the blade during operation would not exceed $251.68 \mathrm{~Hz}$. Thus, first-order bending resonance had the highest occurrence probability and would be the most harmful to the blade. Therefore, this study only considered fatigue damage caused by first-order blade bending resonance.

2.1.3. Blade Model Experimental Verification. We measured the natural frequency of the root-retaining blade using the impulse hammering method and obtained the first-four vibration order shapes using laser scanning.

Six blade specimens were randomly selected, coded as a1-a6. Frequency response for al was obtained by holistically analyzing five pulse simulations following the experimental parameters and settings, as shown in Figure 3, where response peaks noted blade natural frequencies. Moreover, frequency response curves of a $2-\mathrm{a} 6$ are basically the same as that of a1.

According to the frequency response curve of each blade, the first-four order natural frequencies were collected, as shown in Table 4.

Table 5 compares simulation and experimental results and mean error probability for the natural frequencies for all blades $=2.8 \%$ on average, with maximum $<6 \%$.

Having defined the natural frequencies, multiple measurement points were fixed on the leaf body and the exciter frequency adjusted to the natural rates to realize resonance at each stage of the blade. We used a Polytec laser vibrometer to detect displacement response and identify the vibration
TABLE 5: Comparative analysis of natural frequencies.

\begin{tabular}{lcccc}
\hline Order & $\begin{array}{c}\text { The first } \\
\text { order }\end{array}$ & $\begin{array}{c}\text { The second } \\
\text { order }\end{array}$ & $\begin{array}{c}\text { The third } \\
\text { order }\end{array}$ & $\begin{array}{c}\text { The fourth } \\
\text { order }\end{array}$ \\
\hline $\begin{array}{l}\text { Simulation } \\
\text { value }(\mathrm{Hz})\end{array}$ & 251.68 & 873.58 & 1400.8 & 1967.9 \\
$\begin{array}{l}\text { Experimental } \\
\text { average value }(\mathrm{Hz})\end{array}$ & 249.28 & 855.99 & 1354.83 & 1851.06 \\
\begin{tabular}{l} 
Relative error \\
\hline
\end{tabular} & $0.95 \%$ & $2.01 \%$ & $3.28 \%$ & $5.94 \%$ \\
\hline
\end{tabular}

TABle 6: Blade disc geometric description.

\begin{tabular}{lc}
\hline Blade disc & Geometric size \\
\hline Inner diameter $(\mathrm{mm})$ & 220 \\
Outer diameter $(\mathrm{mm})$ & 354 \\
Thickness $(\mathrm{mm})$ & 30 \\
\hline
\end{tabular}

type. Experimental and model results were consistent, verifying that the blade modeling was accurate and reliable.

2.2. Combined Fatigue Test. The blade disc system, i.e., the compressor rotor system, connects the blades to the disc through pins, with the blades distributed around the edge of the disc. The considered blade disc system included 29 blades with other geometry as shown in Table 6.

During operation, the blade disc system is affected by unbalanced excitation causing the blade to vibrate. Static and vibration analyses of the blade disc system were performed to determine an appropriate test load spectrum, where static analyses locate hazardous points of the blade and vibrational analyses identify dangerous resonance of the system and ideal HCF test frequency.

2.2.1. Blade Disc System Static Analysis. In the case of cumulative fatigue damage, blade cracks usually occur in the region with largest equivalent stress under LCF conditions, which are considered the potential hazardous positions of the blade. Stress distribution patterns will be affected by engine design speed (3500 and 11,320 rpm minimum and maximum, respectively) and velocity amplitude. Boundary conditions were defined around the center axis of the disc, fixing and constraining displacements of all nodes on the inner ring, i.e., displacement $=0$. We applied surface-surface contact laws between the disc and the blade, where the disc was the target and the blade the contact body. Rotational speeds were set at 3500, 4500, 5500, 6500, 7500, 8500, 9500, 10,500, and $11,320 \mathrm{rpm}$. Maximum stress position and stress distribution were derived using nonlinear static analysis, and the effect of increasing speed loading on stress distribution was investigated.

The results showed similar stress distribution under the different centrifugal speed loads. The hazardous position with maximum stress was preliminarily located in the central plate lug joints and earring inner, as shown in Figure 4. Maximum principal stress $=592 \mathrm{MPa}$ when speed was maximum $(11,320 \mathrm{rpm})$. 


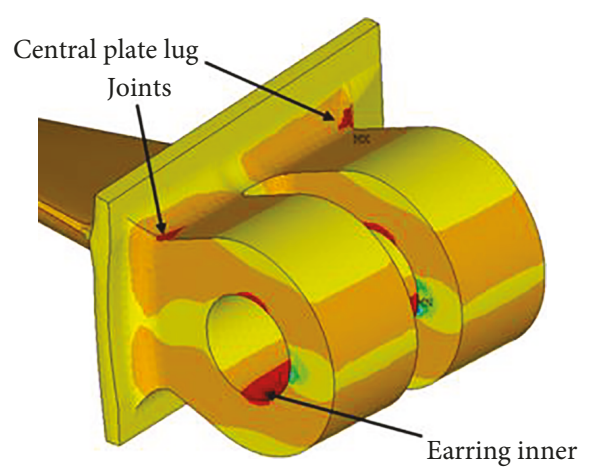

FIgURe 4: Local maximum stress of the blade.

2.2.2. Blade Disc System Vibration Analysis. The blade disc system undergoes centrifugation as it rotates, which increases blade bending stiffness and changes resonance frequencies from those in the stationary state. Therefore, vibrational frequencies change with rotational speed. The dynamic blade curve can be obtained by fitting the relationship between rotational speed and vibration frequencies.

The dangerous blade disc resonance speed was identified as $8425.51 \mathrm{rpm}$, and the first order bending vibration state $(567.29 \mathrm{~Hz})$ from static analysis is shown in Figure 5.

Single-blade vibration characteristics are meaningless and should not be used as the basis for HCF vibration conditions because practical situations almost invariably incorporate multiple coupled blade disc systems. Therefore, we considered coupled vibration characteristics of the system and its resonance frequency to derive the HCF conditions of the blade. If the first-order bending resonance of the system is likely to occur, we can determine the first-order $\mathrm{f}$ requency $=251.68 \mathrm{~Hz}$ of the blade equals to the high cycle vibration frequency, that is, the condition of the resonance failure frequency of the HCF in the CCF test.

2.2.3. Load Spectrum Derivation. We considered the relationship between stress $(S)$ and total fatigue life $(N)$ to investigate the load spectrum, covering pure LCF, pure HCF, and CCF conditions. A CCF test load spectrum was designed based on the typical service loading for an aircraft engine, as shown in Figure 6. The major low-frequency cycle had a trapezoidal stress wave with a $14.59 \mathrm{~s}$ period and included two short blocks and one long block, corresponding to climbing, cruising, and landing, i.e., typical aircraft flight. High-frequency vibration overlaid the low frequency loading, simulating resonance.

Vibration analysis of the blade disc system showed it was prone to first-order resonance and the sinusoidal excitation signal frequency $f_{\mathrm{H}}=251.68 \mathrm{~Hz}$. Similarly, the determined loading frequency ratio was consistent with the design requirements of low $T_{\mathrm{L}}=8250$ cycles and high $T_{\mathrm{H}}=3 \times 10^{7}$ blade cycle life. By calculation, the test frequency ratio is $R_{\mathrm{f}}=3636$ and low cycle loading frequency is $f_{\mathrm{L}}=0.067 \mathrm{~Hz}$.

Because maximum speed, $n_{\max }=11,320 \mathrm{rpm}$, was constant, LCF load, $\sigma_{\mathrm{L}}$, was also constant, as shown in Table 7. Compared with the resonant failure of the blade, forced vibration induced by aerodynamic force is another cause of blade failure. The aerodynamic force mainly manifests as flow pressure on the exhaust side of the blade, defined as approximately $10 \%$ of the maximum centrifugal force [25]. However, the aerodynamic effect should not be considered when CCF of the blade is studied independently. Therefore, we modified the low cycle load to $28,255 \mathrm{~N}$, which was also the LCF test load.

\subsection{Combined Fatigue Test Rig}

2.3.1. Test Rig Design. Traditional HCF test rigs do not accurately represent CCF effects generated in compressor blades. Traditional HCF tests also only considered uniaxial machines, applying both steady and alternating loads along the major specimen axis. The purpose of the CCF test system design core is to optimally simulate actual working conditions using the test rig, and efficient reproduction of the specific blade damage location and failure mechanisms. To simulate LCF and HCF, the designed test rig was divided into LCF and HCF loading devices, as shown in Figure 7.

This design could also be used for pure LCF test. Figure 8 shows the three-dimensional test rig.

The actual test rig is shown in Figure 9. Selecting an appropriate monitoring point and accurately controlling the stress is critical. Because the blade hazardous position is closely connected to the device design, test monitoring points are usually chosen at the blade tip. However, due to severe vibration at the blade tip, the measurement location time domain displacement curve changes exceptionally irregularly, making it difficult to measure amplitude accurately. Therefore, we must select a different monitoring point. Vibration stress was transmitted through the exciting rod to the exciting point and only acts indirectly on the blade hazardous position. The amplitude at the exciting point is difficult to measure. Hence, we measured the amplitude at the hazardous position using the laser vibrometer and assumed these amplitudes were equal. So we finally set the monitoring point at the center of the internal fixture surface, as shown in Figure 10, because the time domain displacement curve for this location was sinusoidal with relatively small amplitude.

\subsubsection{The Function of the Test Rig Realization. Hydraulic} loading equipment can simulate centrifugal forces and balance the stress on the assessed blade part to the static stress for the selected engine rotating speed, producing tension on the steel cable along the radial direction of the blade. Figure 9 shows the loading force was controlled by an electrohydraulic servo valve to meet the LCF requirements. Low cycle load was also monitored by the sensor.

The HCF loading device mainly consisted of the shaker, signal generator, and exciting rod. Under vibration, the excitation signal passes through the exciting rod to the blade, forcing the corresponding position to vibrate in the vertical direction of the blade. Thus, the vibration amplitude of the blade hazardous position varies with the HCF amplitude required by the test, which realizes CCF loading.

2.3.3. Vibration Stress and Amplitude Relationship. Transient thermal responses under seven different amplitudes were 


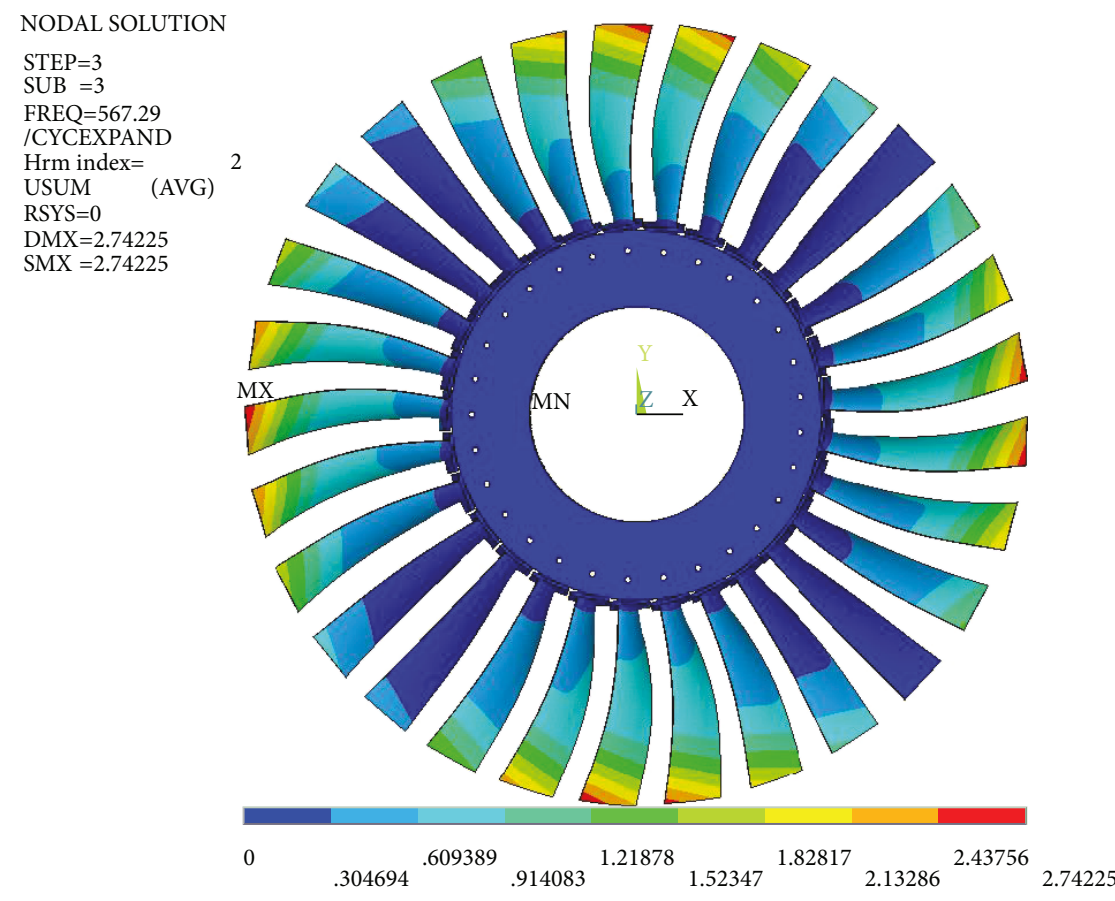

Figure 5: Static analysis of the blade disc system.

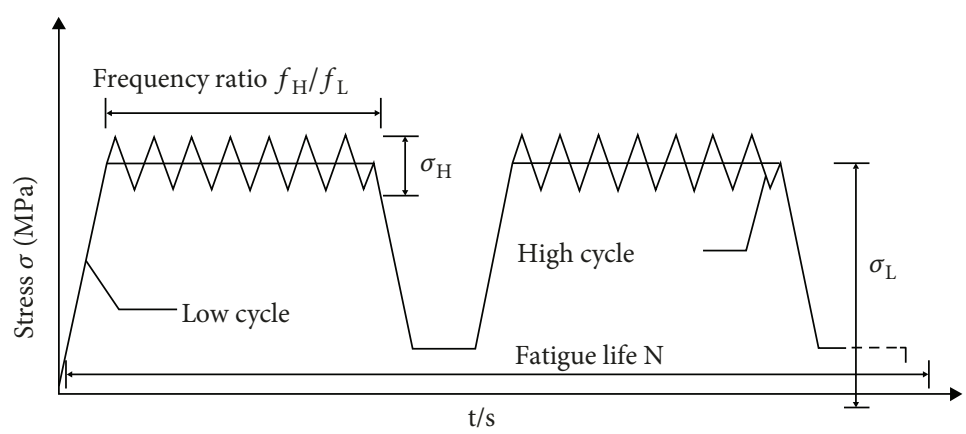

FIgURE 6: Load spectrum of the combined fatigue test.

TABLE 7: LCF load results.

\begin{tabular}{lcc}
\hline $\begin{array}{l}\text { Maximum rotating } \\
\text { speed }(\mathrm{rpm})\end{array}$ & $\begin{array}{c}\text { LCF load simulation } \\
\text { value }(\mathrm{N})\end{array}$ & $\begin{array}{c}\text { LCF load modified } \\
\text { value }(\mathrm{N})\end{array}$ \\
\hline 11,320 & 25,686 & 28,255 \\
\hline
\end{tabular}

calculated, and the average value was obtained. The statistical results are shown in Table 8.

The monitoring point amplitudes were continuously controlled by the shaker at 40, 50, 60, 70, 80, 90, and $100 \mu \mathrm{m}$, with corresponding measured amplitudes 47.49, 59.58, 71.66, 71.66, 83.74, 95.82, 107.91, and $119.99 \mu \mathrm{m}$, respectively, as shown in Table 8 . Vibration amplitude and stress were fitted using the least squares method, as shown in Figure 11.

Vibration stress and amplitude are both linear. Therefore, the exponential can be expressed as

$$
\sigma=1.045 \times A+70.04
$$

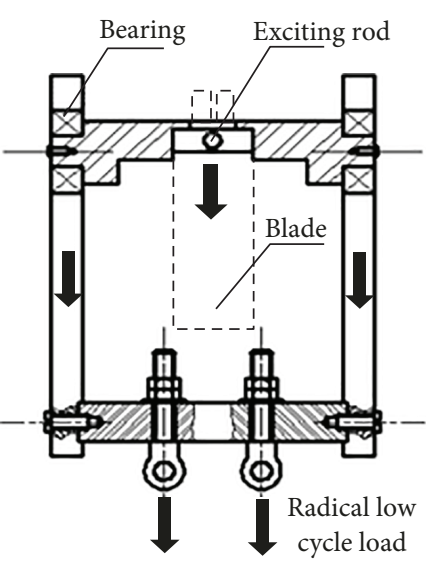

(a) LCF load path on radial direction in main view

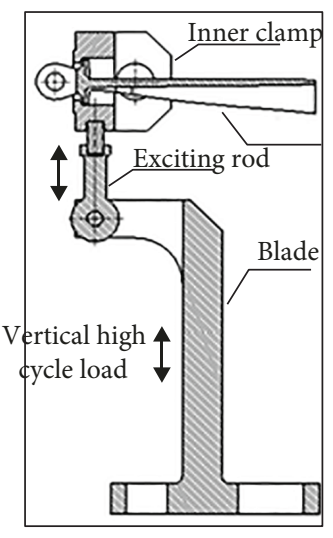

(b) HCF Load path on vertical direction in side view
FIgURE 7: Load path of the CCF. 


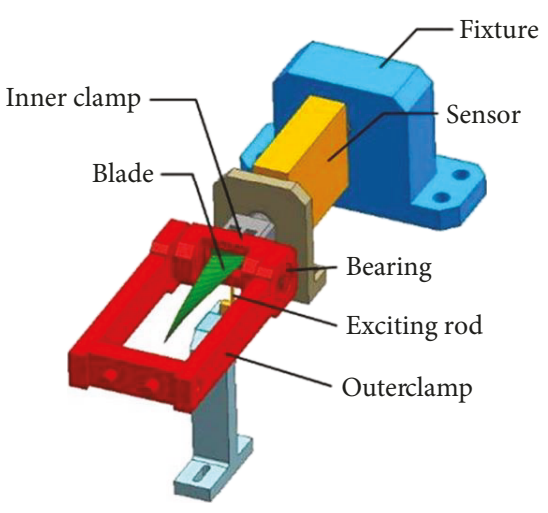

Figure 8: Combined fatigue test rig.

where $\sigma$ is the vibration stress and $A$ is the monitoring point amplitude. Therefore, vibration stress that cannot be measured directly needs to be converted to amplitude, because the vibration stress amplitude of the blade hazardous location at any amplitude can be determined.

According to the numerical results, we have explored the CCF test conditions, formulated the load spectrum, and realized the CCF test rig. Before studying the effects of different vibration stresses on LCF life under the low cycle load holding constant, we must first determine the correctness of the numerical results and the rationality of the combined test design based on fracture investigations of the test blade and study the relationship between vibration stress and amplitude. Taking into account these analyses, in the following studies, we conduct fracture tests and explore the effects of CCF on blade fatigue life.

\section{Results and Discussion}

3.1. Fractographic Investigations. Fracture characteristics changed under combined cycling with strong interaction among LCF, HCF, and crack initiation. Figures 12-17 show the major manifestations, which validate the CCF test system design.

A visual crack appeared at the root of the blade, corresponding to the static results of the hazardous location. Processed fracture patterns were applied to the specimens and scanned using SEM.

3.1.1. Fracture Behavior under CCF. Figures 13 and 14 show fracture modes indicating the crack initiation and propagation mechanism under CCF for different loading conditions. Figure 13 shows that region 1 is the crack source, region 2 is the high loading fracture zone, region 3 is the low loading fracture zone, and region 4 is the fracture morphology formed during the process. The fracture surface of the high cycle zone is smooth, while in the lower region it is rough.

Figure 14 shows grain boundary facets with cracks originating at grain boundary junctions alongside transgranular fatigue cracks, indicating build-up of time-dependent creep damage. The crack surface has an uneven oxide film, probably due to the prolonged exposure of the fracture in the air, which also affects the SEM analysis. Crack initiation was mostly transgranular, indicating fatigue was the predominant contributor to the overall damage. A similar trend was also evident for crack propagation modes under LCF, particularly when the blades were subjected to lower stabilized cyclic stress.

3.1.2. Fracture Analysis under LCF [26]. Figures 15 and 16 show the low expansion region. There is a multisource or linear-source fracture in the low cycle fatigue extended region, with occasional small dimples found nearby the fatigue source area. The fatigue band spacing of the extended region is larger, and separation distance generally exceeded micrometers.

There was obvious transgranular phenomenon and cavity nucleation. Fractures were predominantly along the grain boundaries, indicating they were caused by typical tensile forces. Intergranular cracks are developed through cavity formation at the grain boundaries due to high local stress concentration caused by grain boundary sliding. With increasing scanning time, transgranular initiated cracks were characterized by striations with secondary cracks present along near-surface regions, whereas stress-dependent tensile ratcheting became more prominent in the form of dimples (ductile rupture) towards the interior (Figure 15).

Thus, the predominance of tensile ratcheting in the crack propagation phase highlighted two failure modes: timedependent intergranular fracture and stress-dependent ductile rupture. This process was also facilitated by impingement of slip bands at the grain boundary. However, at very high stresses, particles within a grain became decohered at the particle-matrix interface due to tensile ratcheting, resulting in dimple formation that eventually leads to ductile failure. Hence, these two processes are competitive and governed by both the applied stress and the dwell time. The predominance of compressive ratcheting due to the lower stabilized cyclic stress prevented fast damage accumulation as occurred when stress-dependent mechanisms were highly active, providing sufficient time for cavity nucleation at grain boundaries (Figure 16).

3.1.3. Fracture Analysis under HCF. Figure 17 shows selected portions of the fracture surface at higher magnification to assess HCF damage, including transverse fatigue striation and edge tearing in bright linear zones. Thus, fracturing was caused by transgranular expansion. The cross section between the fatigue source and extension area was bright and smooth, with many light fatigue arc curves in the fracture diagram. Furthermore, HCF striations were also directly ahead of grain boundary facets, indicating that minute $\mathrm{HCF}$ cracks are linked with intergranular cracks [26].

During the final failure, the uncracked ligaments between the arrested cracks presumably got torn off by shearing, forming extensive tear zones. Moreover, these striations tended to link with adjoining grain boundary facets, confirming that HCF damage acted as a channel to link LCF with crack source zones.

The cracks could not advance further by coalescing because a highly stabilized cyclic stress would lead to ruptures, as evidenced by extensive dimples. Thus, HCF damage 


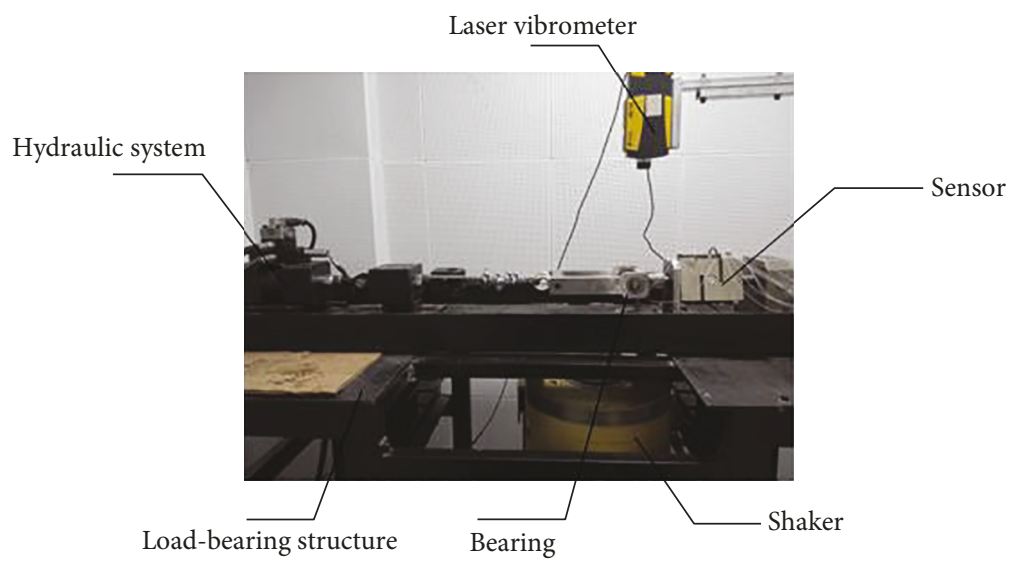

Figure 9: Assembly diagram of blade fixture system.

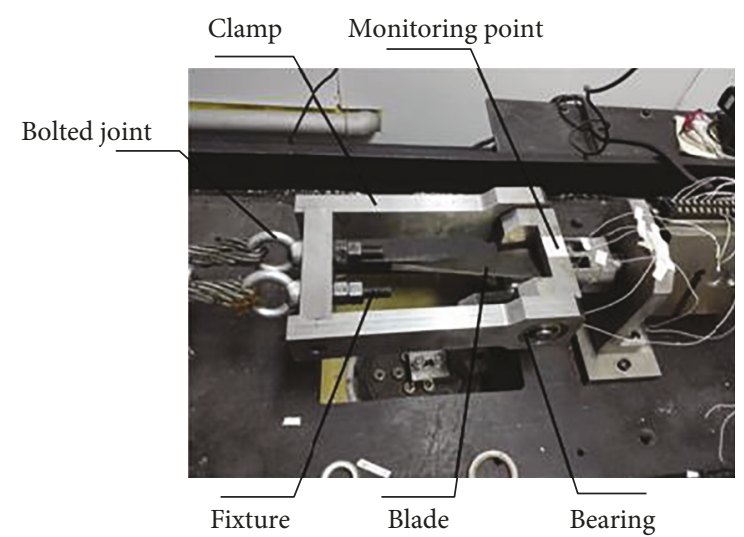

FIGURE 10: Vibration monitoring point diagram.

TABLE 8: Vibration stress and amplitude relationship of blade hazard position.

\begin{tabular}{lcc}
\hline $\begin{array}{l}\text { Amplitude of exciting } \\
\text { point }(\mu \mathrm{m})\end{array}$ & $\begin{array}{c}\text { Amplitude of } \\
\text { monitoring point }(\mu \mathrm{m})\end{array}$ & $\begin{array}{c}\text { Vibration } \\
\text { stress }(\mathrm{MPa})\end{array}$ \\
\hline 47.49 & 40 & 112.03 \\
59.58 & 50 & 122.27 \\
71.66 & 60 & 133.64 \\
83.74 & 70 & 141.59 \\
95.82 & 80 & 152.23 \\
107.91 & 90 & 166.28 \\
119.99 & 100 & 173.9 \\
\hline
\end{tabular}

in such cases was negligible and might not have affected the final failure. When LCF damage is negligible and timedependent damage is predominant in terms of the extensive intergranularity of fracture, HCF plays a similar role as revealed from the presence of striations within grain boundary facets which originated mostly from HCF cycling.

3.2. Fatigue Life Test. Through fracture results, we verified the rationality of the design of the experimental system, which can be used as a study of the combined fatigue effect of blades. Pure LCF and CCF tests were then performed to identify fatigue life characteristics.

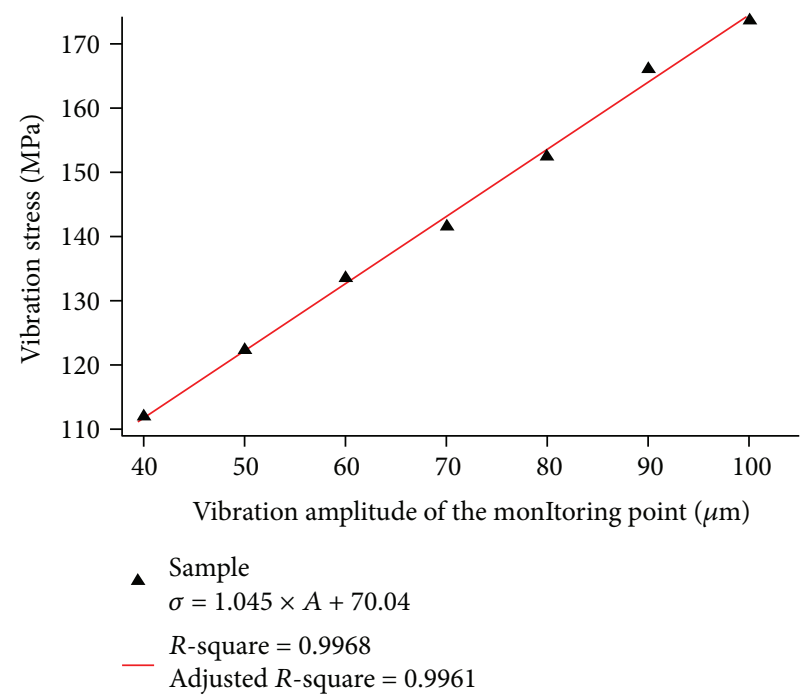

FIGURE 11: Relationship between vibration stress and amplitude.

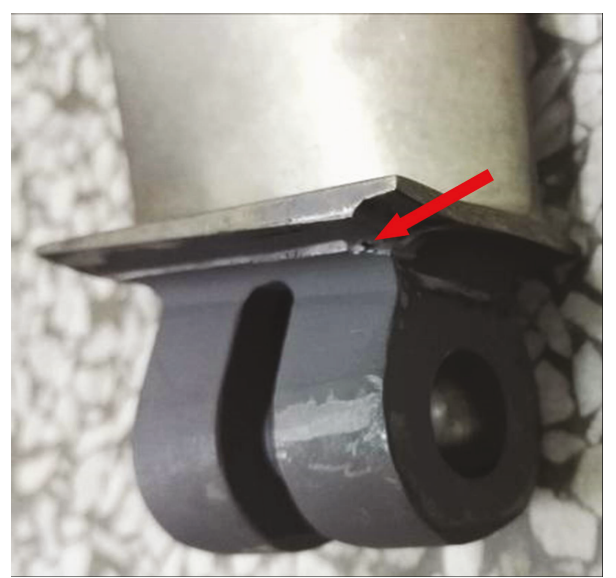

FIGURE 12: Fracture inspection place of the blade.

3.2.1. Pure Low Cycle Fatigue Test and Result Analysis. Table 9 shows pure low cycle fatigue life test results for constant low cycle load $(28,225 \mathrm{~N})$ on seven blade specimens. 


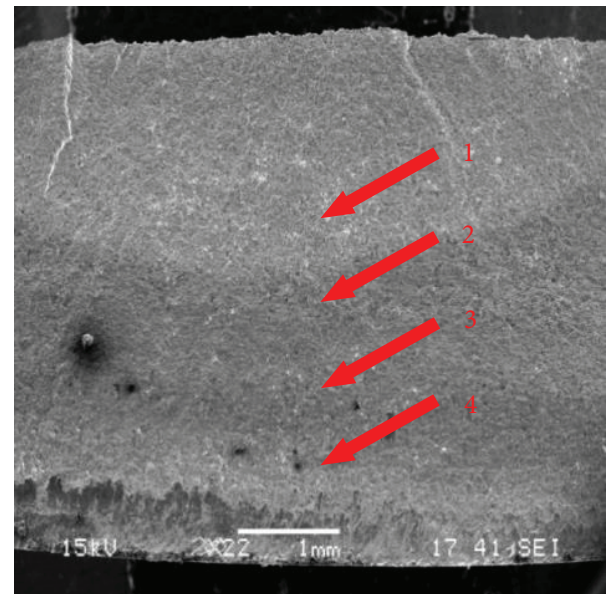

FIGURE 13: SEM fracture image.

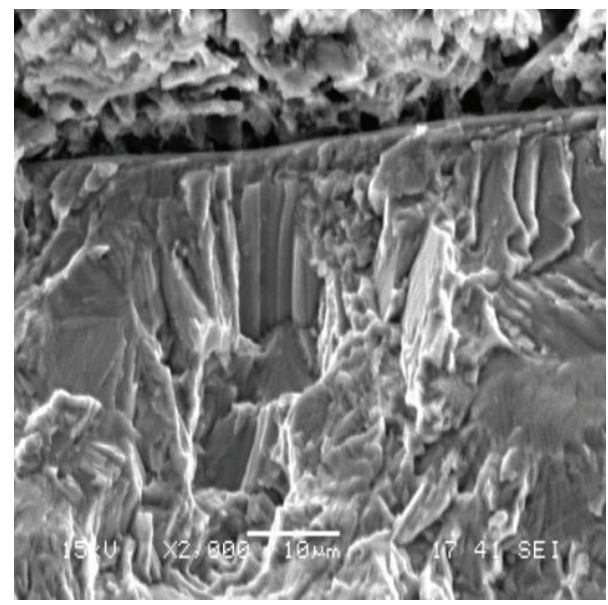

FIGURE 14: SEM fracture image, 2000x magnification.

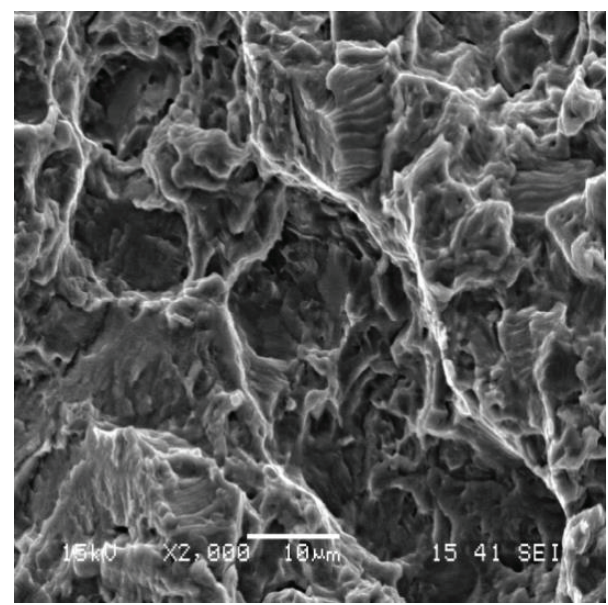

Figure 15: SEM LCF area, 2000x magnification.

3.2.2. Combined Fatigue Test and Result Analysis. Seven blades were selected for CCF tests under seven stress levels with constant low cycle load to compare with the pure LCF test and highlight HCF effects on fatigue life. Monitoring point amplitudes were controlled to $40,50,60,70,80,90$,

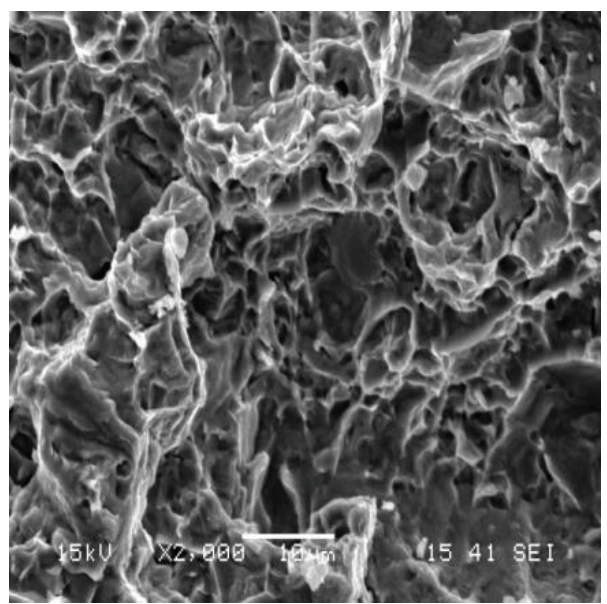

FIGURE 16: SEM cavity nucleation, 2000x magnification.

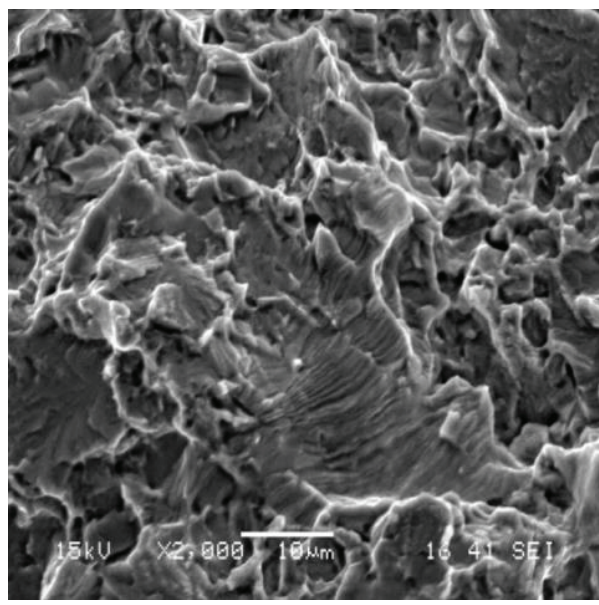

FIGURE 17: SEM HCF area, 2000x magnification.

TABle 9: Pure low cycle fatigue test results.

\begin{tabular}{lcc}
\hline Serial number & Low cycle load $(\mathrm{N})$ & LCF life (cycle) \\
\hline 1 & 28,255 & 33,372 \\
2 & 28,255 & 45,361 \\
3 & 28,255 & 36,458 \\
4 & 28,255 & 53,145 \\
5 & 28,255 & 31,467 \\
6 & 28,255 & 38,473 \\
7 & 28,255 & 41,824 \\
\hline
\end{tabular}

and $100 \mu \mathrm{m}$, respectively, and the outcomes are shown in Table 10.

Table 9 indicates that pure LCF life can exceed 30,000 cycles whereas Table 10 shows that maximum LCF life under combined loading was only 11,241 cycles. The blade fatigue life under pure low cycle loading is much longer than that under the CCF, indicating that blade fatigue life can be significantly reduced by HCF loading and that the higher superimposed vibration stress level can lead to more significant reduction in the LCF fatigue life. 
TABLE 10: HCF/LCF combined test records at different stress levels.

\begin{tabular}{lccccc}
\hline $\begin{array}{l}\text { Serial } \\
\text { number }\end{array}$ & $\begin{array}{c}\text { Amplitude } \\
(\mu \mathrm{m})\end{array}$ & $\begin{array}{c}\text { LCF } \\
\text { load }(\mathrm{N})\end{array}$ & $\begin{array}{c}\text { HCF load } \\
(\mathrm{MPa})\end{array}$ & $\begin{array}{c}\text { LCF life } \\
(\text { cycle })\end{array}$ & $\begin{array}{c}\text { HCF life } \\
(\text { cycle })\end{array}$ \\
\hline 1 & 40 & 28,255 & 112.03 & 11,241 & $40,872,276$ \\
2 & 50 & 28,255 & 122.27 & 10,120 & $36,796,320$ \\
3 & 60 & 28,255 & 133.64 & 9400 & $34,178,400$ \\
4 & 70 & 28,255 & 141.59 & 8675 & $31,542,300$ \\
5 & 80 & 28,255 & 152.53 & 8094 & $29,429,784$ \\
6 & 90 & 28,255 & 166.28 & 7642 & $27,786,312$ \\
7 & 100 & 28,255 & 173.9 & 7270 & $26,433,720$ \\
\hline
\end{tabular}

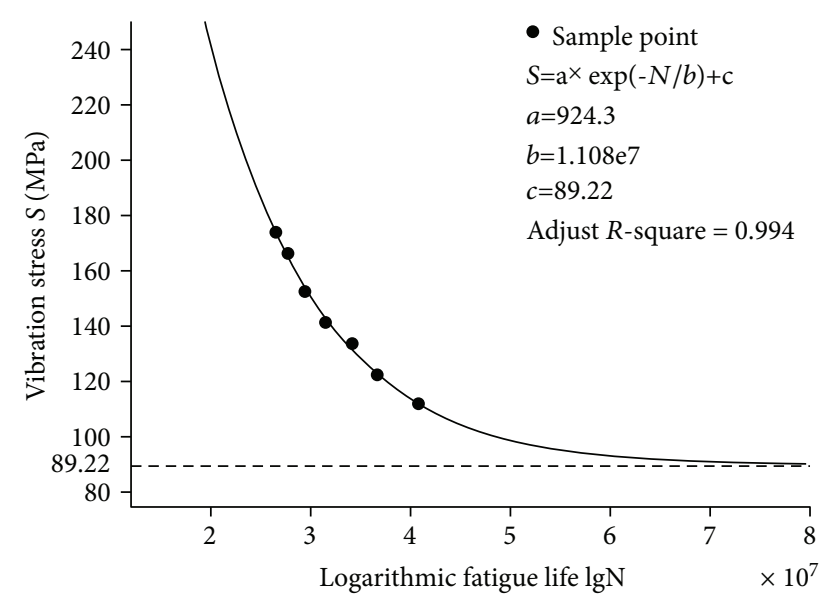

Figure 18: $S$-N curves in linear coordinates.

An overall reduction in CCF life compared with pure LCF was possibly due to HCF effects on fatigue crack propagation rate. The fraction of life spent in propagating a fatigue crack would be reduced but remain significant due to the lower stress range associated with minor cycles, hence significantly reducing total fatigue life. Accelerated crack growth could have been caused by the unloading associated with the LCF part of the CCF waveform, as demonstrated by Fleck and Smith [27] both in steel and aluminium alloy [20].

It is important to study HCF effects on LCF life in CCF testing and blade fatigue characteristics to predict blade life under any stress conditions. These results showed that HCF had a significant effect on LCF life, but the extent of this impact requires further study. So we need to analyze the combined test results in Table 10.

3.2.3. Median Cyclic Stress-Fatigue Life Curve. Fatigue characteristics are usually expressed using the relationship between maximum stress, $S$, and fatigue life, $N$, i.e., the $S-N$ curve, which illustrates the endurance of specimens to changing stresses. Figure 18 shows the fitted $S-N$ curve in linear coordinates.

Vibration stress and fatigue life are exponentially related, as

$$
S=a \times \exp \left(-\frac{N}{b}\right)+c
$$

and the fitted result shows that $a=924.3 \mathrm{MPa}, b=1.108 \times$ $10^{7}$ cycles, and $c=89.22 \mathrm{MPa}$, where $c$ is the vibration stress threshold. Hence, the HCF vibration load had no significant effect on blade LCF life when vibration stress $\leq 89.22 \mathrm{MPa}$. Stress exceeded this threshold when we tested under maximum stress conditions, creating significant plasticity such that time spent initiating fatigue cracks was relatively short.

\section{Conclusion}

This study developed a CCF test system to investigate blade failure caused by centrifugal force and vibration combined. This combination more accurately represented practical conditions the blade would be subjected to in service. Hence, it becomes apparent that the previous and current design methods for blade material based upon HCF performance remain valid, because the original conservatism was retained. The fracture analyses have verified the rationality and feasibility of the designed combined fatigue test rig. Also, the life characteristics showed that the blade LCF life can be significantly reduced by the HCF loading. Increased vibration stress could lead to a more significant blade fatigue life reduction.

\section{Data Availability}

The blade mode simulation data and nature frequency data and pure low cycle fatigue test data and HCF/LCF combined test data at different stress levels involved in Tables 2, 3, 6, and 7 used to support the findings of this study have not been made available because the experimental data involved in the paper are all obtained based on my own designed experiment and need to be kept confidential.

\section{Conflicts of Interest}

The authors declare that they have no conflicts of interest.

\section{Acknowledgments}

The author would like to thank the anonymous reviewers for their detailed and constructive comments and suggestions. The support from the National Natural Science Foundation of China (Grant No. 51175510) is also gratefully acknowledged.

\section{References}

[1] N. S. Xi, P. D. Zhong, H. Q. Huang, H. Yan, and C. H. Tao, "Failure investigation of blade and disk in first stage compressor," Engineering Failure Analysis, vol. 7, no. 6, pp. 385-392, 2000.

[2] N. Hou, Z. Wen, Q. Yu, and Z. Yue, “Application of a combined high and low cycle fatigue life model on life prediction of SC blade," International Journal of Fatigue, vol. 31, no. 4, pp. 616-619, 2009.

[3] Y. K. Zhang, R. X. Zhou, S. X. Guo, B. L. Shang, and W. T. Jia, "Design of compound fatigue test system of compressor blade and fatigue life analysis," Journal of Aerospace Power, vol. 32, no. 12 , pp. 2880-2887, 2017. 
[4] Y. Huang, Y. Liu, and W. Liu, "Analysis of structural-dynamic random reliability sensitivity based on the criteria of fatigue cumulative damage," Aircraft Design, vol. 30, no. 2, pp. 5-9, 2010.

[5] A. Fatemi and N. Shamsaei, "Multiaxial fatigue: an overview and some approximation models for life estimation," International Journal of Fatigue, vol. 33, no. 8, pp. 948-958, 2011.

[6] S. Liu, C. Liu, Y. Hu, S. Gao, Y. Wang, and H. Zhang, "Fatigue life assessment of centrifugal compressor impeller based on FEA," International Journal of Fatigue, vol. 60, pp. 383-390, 2016.

[7] X. Y. Luo, R. G. Zhao, and W. He, "Analysis on low cycle fatigue properties and fractography of TC25 titanium alloy," Chinese Journal of Solid Mechanics, vol. 32, pp. 145-149, 2011.

[8] X. Yuan and S. Li, "Research and development of methods for the prediction of fatigue life," Aeronautical Manufacturing Technology, vol. 12, pp. 80-84, 2001.

[9] A. Sarkar, A. Nagesha, P. Parameswaran, R. Sandhya, K. Laha, and M. Okazaki, "Investigation of cumulative fatigue damage through sequential low cycle fatigue and high cycle fatigue cycling at high temperature for a type $316 \mathrm{LN}$ stainless steel: life-prediction techniques and associated mechanisms," Metallurgical and Materials Transactions A, vol. 48, no. 3, pp. 953964, 2017.

[10] H. O. Fuchs, D. V. Nelson, and T. L. Toomay, Fatigue Under Complex Loading, Society of Automotive Engineers, Warrendale, PA, USA, 1977.

[11] T. Nicholas, "Critical issues in high cycle fatigue," International Journal of Fatigue, vol. 21, pp. 221-S231, 1999.

[12] M. A. Moshier, T. Nicholoas, and B. M. Hillberry, High Cycle Fatigue Threshold in the Presence of Naturally Initiated Small Surface Cracks, American Society for Testing and Materials, West Conshohochen, PA, USA, 2002.

[13] C. Dungey and P. Bowen, "The effect of combined cycle fatigue upon the fatigue performance of Ti-6Al-4V fan blade material," Journal of Materials Processing Technology, vol. 153154, pp. 374-379, 2004.

[14] R. Wang, C. Cho, and J. Nie, "Combined fatigue life test and extrapolation of turbine disk mortise at elevated temperature," Journal of Engineering for Gas Turbines and Power, vol. 127, no. 4, pp. 863-868, 2005.

[15] C. Schweizer, T. Seifert, B. Nieweg, P. von Hartrott, and $\mathrm{H}$. Riedel, "Mechanisms and modelling of fatigue crack growth under combined low and high cycle fatigue loading," International Journal of Fatigue, vol. 33, no. 2, pp. 194-202, 2011.

[16] B. Powell, T. Duggan, and R. Jeal, "The influence of minor cycles on low cycle fatigue crack propagation," International Journal of Fatigue, vol. 4, no. 1, pp. 4-14, 1982.

[17] D. Gelmedin and K.-H. Lang, "Fatigue behaviour of the superalloy IN 713C under LCF-, HCF- and superimposed LCF/ HCF-loading," Procedia Engineering, vol. 2, no. 1, pp. 13431352, 2010.

[18] Z. Mazur, A. Hernández-Rossette A, and J. Porcayo-Calderón, "Fatigue investigation of the $69 \mathrm{MW}$ gas turbine of a combined cycle unit," in ASME Turbo Expo 2010: Power for Land, Sea and Air, pp. 481-489, Glasgow, UK, 2010.

[19] S. A. Namjoshi and S. Mall, "Fretting behavior of Ti-6Al-4V under combined high cycle and low cycle fatigue loading," International Journal of Fatigue, vol. 23, pp. 455-461, 2001.

[20] M. Naeem, R. Singh, and D. Probert, "Implications of engine deterioration for a high-pressure turbine-blade's low-cycle fatigue (LCF) life-consumption," International journal of Fatigue, vol. 21, no. 8, pp. 831-847, 1999.

[21] J. Ding, R. F. Hall, J. Byrne, and J. Tong, "Fatigue crack growth from foreign object damage under combined low and high cycle loading. Part I: experimental studies," International Journal of Fatigue, vol. 29, no. 7, pp. 1339-1349, 2007.

[22] L. Mendia, F. J. Estensoro, C. Mary, and F. Vogel, "Effect of combined cycle fatigue on Ti6242 fatigue strength," in The 11th International Conference on the Mechanical Behavior of Materials (ICM11), pp. 1809-1814, Como, Italy, 2011.

[23] Committee for Practical Handbook of Engineering Materials, "Magnesium Alloys and Titanium alloys," in Practical Handbook of Engineering Materials: Aluminum alloys, Standards Press of China, 1989.

[24] Beijing Aviation Materials research institute, Material Data Sheet of Aircraft Engine Design, China Aviation Engine Company, 1992.

[25] J. Hong, D. Y. Zhang, and L. L. Ghen, "Review on investigation of high cycle fatigue failures of the aero engine blade," Journal of Aerospace Power, vol. 24, no. 3, pp. 652-661, 2007.

[26] A. Sarkar, M. Okazaki, A. Nagesha, P. Parameswaran, R. Sandhya, and K. Laha, "Mechanisms of failure under low cycle fatigue, high cycle fatigue and creep interactions in combined cycling in a type 316LN stainless steel," Materials Science and Engineering: A, vol. 683, pp. 24-36, 2017.

[27] N. A. Fleck and R. A. Smith, "A discussion of mechanisms for acceleratedand retarded fatigue crack growth," in Sixth International Conference on Fraction, pp. 1832-1829, Delhi, India, 1984. 


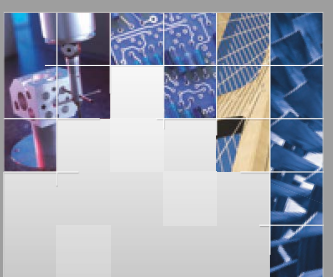

\section{Enfincering}
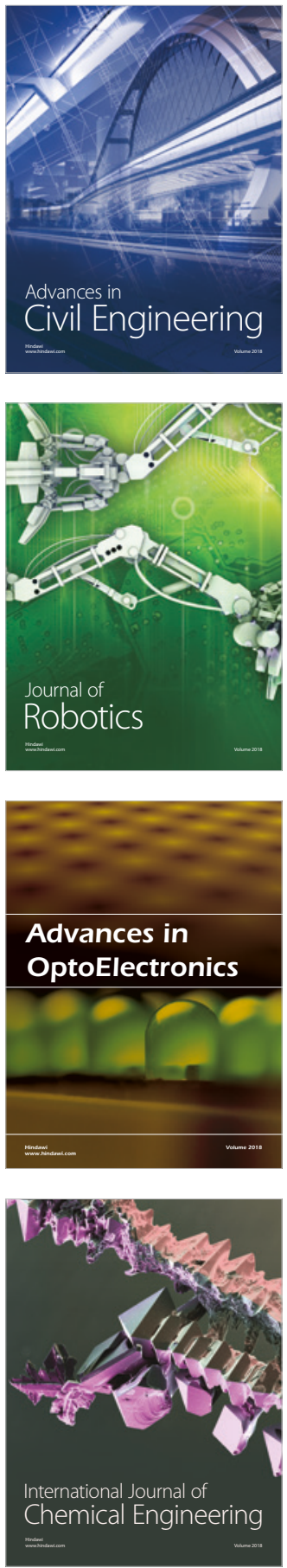

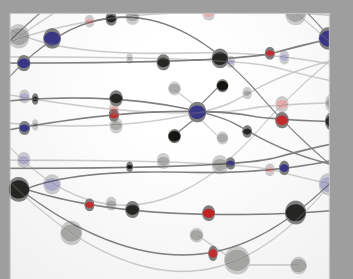

\section{Rotating \\ Machinery}

The Scientific World Journal

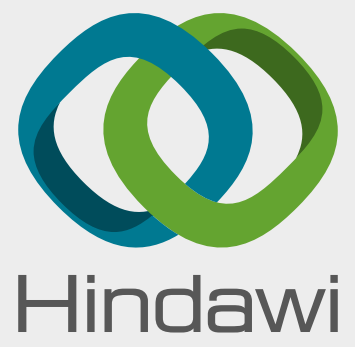

Submit your manuscripts at

www.hindawi.com
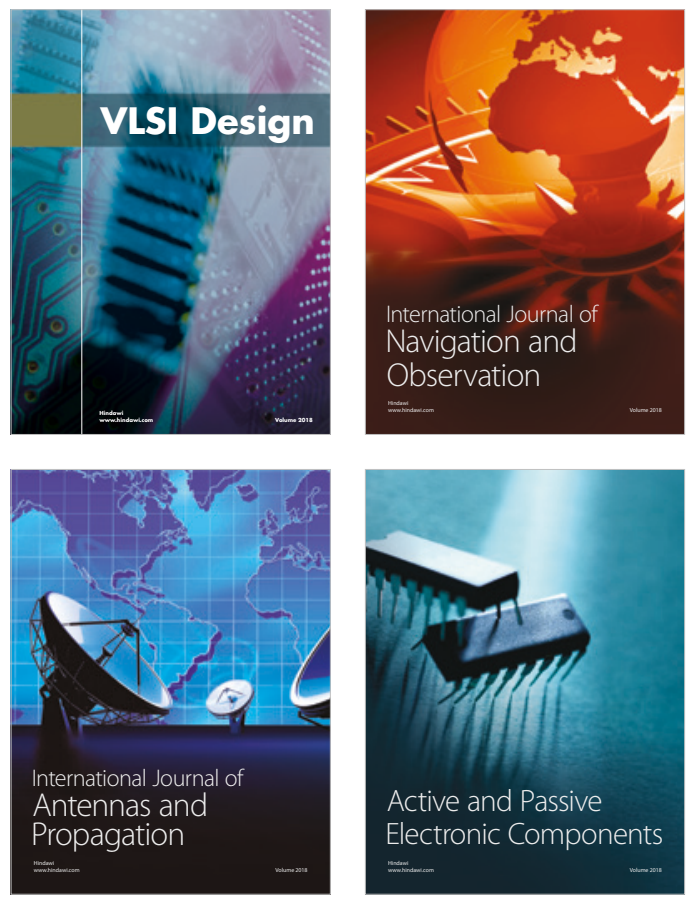
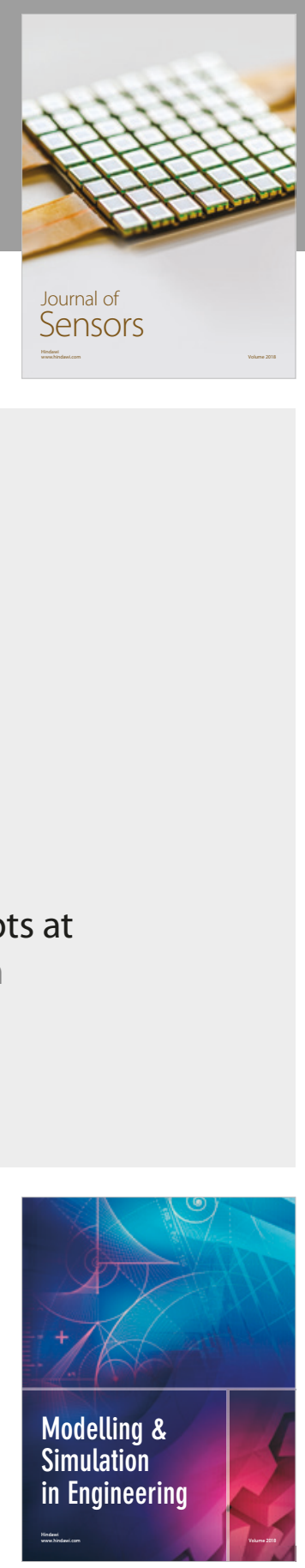

\section{Advances \\ Multimedia}
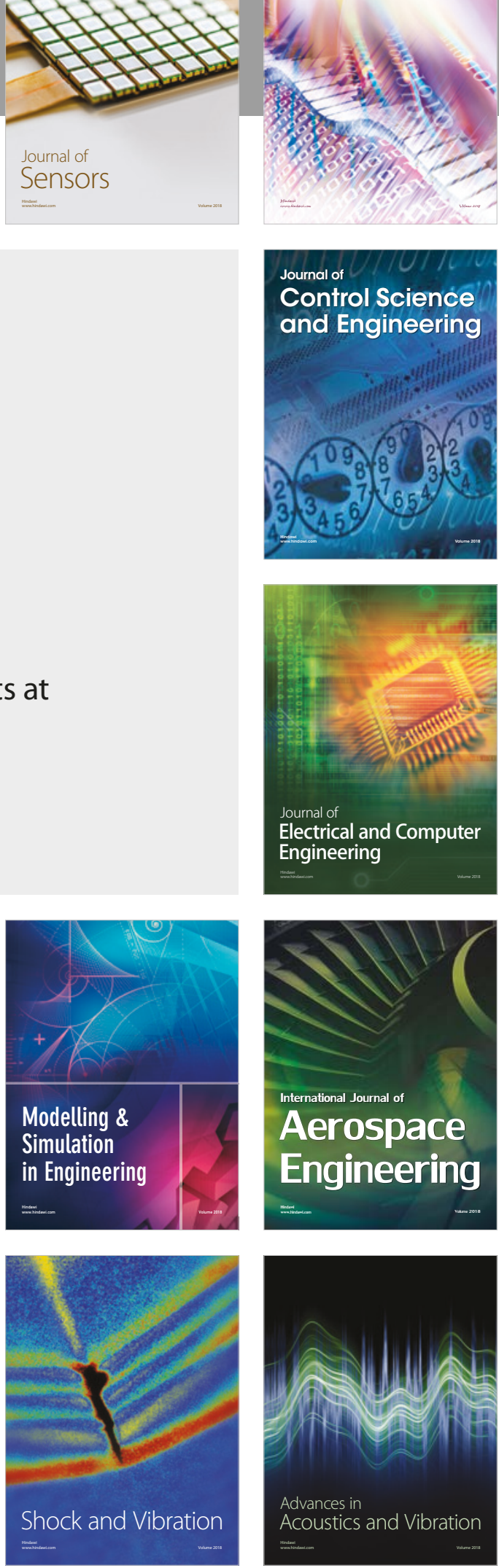